\title{
Formulação e implementação de políticas públicas saudáveis: desafios para 0 planejamento e gestão das ações de promoção da saúde nas cidades
}

\section{Formulation and implementation of healthy public policies: challenges for planning and management of health promotion in the cities}

\section{Carmen Fontes Teixeira}

Doutora em Saúde Pública. Professora do Instituto de Saúde Coletiva da Universidade Federal da Bahia.

E-mail: carmentœufba.br

\section{Resumo}

0 presente artigo contempla, inicialmente, uma breve revisão das noções e conceitos que fundamentam a proposta de elaboração de Políticas Públicas Saudáveis (PPS), discutindo a possibilidade de enriquecimento desta noção à luz do debate conceitual no campo da Saúde Coletiva. Em seguida, trata de apresentar e comentar alguns procedimentos metodológicos que podem ser utilizados no processo de formulação e implementação de "políticas públicas saudáveis”, desenvolvendo uma reflexão acerca das possibilidades de utilização do enfoque situacional como eixo do processo de planejamento dessas políticas no âmbito nacional, estadual e, principalmente, municipal. Por último, discute o significado estratégico da institucionalização do planejamento e gestão das PPS tendo em vista a elevação da capacidade de governo das organizações públicas, a descentralização da gestão e a integralidade das práticas diante da complexidade da situação de saúde da população brasileira.

Palavras-chave: Promoção da Saúde; Políticas públicas saudáveis; Enfoque situacional; Planejamento e gestão; Cidades. 
Abstract

This article deals, initially, with a brief review of the notions and concepts which are the foundations of the Healthy Public Policies (HPP), discussing the possibility of enrichment of this notion at the light of the conceptual debate in the field of Collectiona Health. Secondloy, it presents and comments on some methodological procedures that could be used in the process of formulation and implementation of HPP, developing a reflexiona on the possibilities of using the situational approach as the axis for plannning these policies in the national, state, and mainly, municipal level. Finally, it discusses the strategic meaning of the institutionalization of HPP planning and management with a view at enhancing the governance capacity of public organitions, the decentralization of management and the integrality of the practices face the complexity of the health situation of the Brazilian population.

Key Words: Health Promotion; Healthy Public Policies; Situational Approach; Planning and Management; Cities.

\section{Introdução}

A noção de "Políticas Públicas Saudáveis" - PPS apareceu no debate internacional durante a $8^{\text {a Conferên- }}$ cia Internacional de Promoção da Saúde, realizada em Ottawa, Canadá, em 1986, no mesmo ano em que, no Brasil, aconteceu a 8. ${ }^{a}$ Conferência Nacional de Saúde, marco do processo brasileiro de Reforma Sanitária. A Carta de Ottawa, produto da Conferência Internacional, considera a elaboração e implementação de PPS um dos cinco campos de ação social para a promoção da saúde, ao lado da criação de ambientes favoráveis à saúde, reforço da ação comunitária, desenvolvimento de habilidades pessoais e a reorientação do sistema de saúde (Buss, 2003).

A divulgação das idéias e propostas contidas na Carta de Ottawa subsidiou um amplo movimento que se desdobrou nas Conferências ulteriores, realizadas em Adelaide, Austrália (1988), Sundsval, Suécia (1991), Jacarta, Indonésia (1997), na América Latina, inicialmente em Bogotá (1992) e posteriormente em México (2000) e Brasil (2002), as quais consolidaram vários dos conceitos estratégicos da proposta de Promoção da Saúde (Brasil - MS, 2001). O conjunto dessas idéias vem subsidiando a formulação de políticas em vários planos e níveis de governo, em vários países, configurando, entre outros desdobramentos, o movimento em torno da constituição de "cidades saudáveis", estratégia assumida pela OMS em 1984 para fomentar a reorientação da gestão governamental em nível local (Ferraz, 1999).

No Brasil, a difusão das idéias do movimento pela Promoção da Saúde vem ocorrendo em um contexto marcado pela formulação e implementação das propostas oriundas do movimento da Reforma Sanitária, especialmente o processo de construção do SUS, pontuado, nas distintas conjunturas que se configuraram nos últimos 15 anos, pela formulação de políticas, programas e projetos de reforma na organização e gestão das ações e serviços de saúde, em vários níveis de governo. No âmbito desse processo, vem se dando, em algum grau, a incorporação de noções oriundas do debate em torno da criação de ambientes e estilos de vida saudáveis, como ocorreu na concepção do Programa de Combate ao Tabagismo, no Programa de Controle da AIDS, no Programa de Controle da Hipertensão e Diabetes e também se apresenta no debate em 
torno da incorporação de ações de promoção da saúde na área de Saúde da Mulher, do Adolescente, do Idoso e outros grupos prioritários.

Além disso, observa-se a incorporação das propostas da Promoção da Saúde no âmbito do Programa de Saúde da Família, bem como no debate acerca da estruturação do sistema de vigilância da saúde no SUS, quer na área de Vigilância epidemiológica, quer na área de Vigilância ambiental, estruturada no MS ao final dos anos 90 (Teixeira, 2003). Especialmente em nível municipal, a difusão e a incorporação da proposta de criação de "cidades saudáveis" têm sido incorporadas por várias administrações municipais nos últimos anos como Campinas, Curitiba, Fortaleza, entre outras (Akerman, 1997; Junqueira, 1997, 1998; Ribeiro, 1997). Esse processo gerou a acumulação de uma experiência que hoje fundamenta a organização de uma rede de municípios saudáveis em todo o país, iniciativa que conta com o apoio da OPAS e dialoga com um conjunto de entidades, inclusive o CONASEMS (Sperandio, 2004).

Em 1998, o Ministério da Saúde elaborou um projeto de Promoção da Saúde sob responsabilidade da então Secretaria de Políticas de Saúde, a qual foi responsável pelo estímulo e difusão de um conjunto de experiências inovadoras no âmbito do SUS, através da publicação de uma revista específica sobre o tema. No momento atual, o debate sobre a incorporação da Promoção da Saúde na "agenda do SUS" vem sendo conduzido pela Secretaria Executiva do MS, órgão responsável pela articulação de iniciativas um tanto dispersas na instituição.

Assim, pretende-se identificar e coordenar ações que vem sendo desenvolvidas no âmbito da Secretaria de Atenção à Saúde, particularmente o Departamento de Ações Programáticas Estratégicas, na Secretaria de Vigilância da Saúde, particularmente a Coordenação Geral de Vigilância Ambiental, na Secretaria de Gestão Participativa, especialmente o Departamento responsável pelas ações de Educação Popular em saúde, e na Secretaria de Ciência e Tecnologia, que através do DECIT incorporou a promoção da Saúde na Agenda de Prioridades de Pesquisa na área (MS, 2004).

Em recente Seminário promovido pelo MS com a finalidade de discutir o tema e as possibilidades de incorporação e desenvolvimento dessas propostas, defendemos a idéia de que o debate em torno da Pro- moção da Saúde pode adquirir um significado estratégico, na medida em que se constitua como um dos referenciais que ajudem a retomar e atualizar o conjunto de propostas do projeto de Reforma Sanitária, cujo escopo ultrapassa o processo de construção do SUS e pressupõe a formulação e implementação de políticas econômicas e sociais que tenham como propósito a melhoria das condições de vida e saúde dos diversos grupos sociais, de modo a reduzir as desigualdades sociais, promovendo equiidade e justiça no acesso às oportunidades de trabalho, melhoria dos níveis de renda e garantia das condições de segurança e acesso a moradia, educação, transporte, lazer e serviços de saúde.

Tanto em nível nacional, quanto estadual e municipal, a proposta de avançar no enfrentamento desse desafio exige, além de vontade política por parte dos dirigentes de entidades governamentais e não-governamentais, o desenvolvimento de uma visão estratégica capaz de perceber as implicações decorrentes da adoção das idéias e propostas contidas no corpo doutrinário da Promoção da Saúde, especialmente no que se refere à mudança de enfoque no processo de formulação e implementação de políticas e à mudança na organização e no conteúdo das práticas de saúde.

Partindo do pressuposto de que é possível estabelecer uma leitura crítica e reconstrutiva dessa proposta à luz do debate atual no campo da Saúde Coletiva, especialmente no que diz respeito à reflexão acerca dos determinantes sociais do "complexo saúde-doença-cuidado" (Almeida Filho, 1999) e das perspectivas teórico-metodológicas do planejamento e gestão de ações de promoção da saúde no âmbito governamental, o presente artigo contempla, inicialmente, uma breve revisão das noções e conceitos que fundamentam a proposta de elaboração de Políticas Públicas Saudáveis - PPS, tentando estabelecer o que significa qualificar uma política pública como "saudável".

Em seguida, trata de apresentar e comentar alguns procedimentos metodológicos que podem ser utilizados no processo de formulação e implementação de "políticas públicas saudáveis", resgatando e ampliando uma reflexão acerca das possibilidades de utilização do enfoque situacional no planejamento de ações intersetoriais para a promoção da saúde no âmbito municipal (Teixeira e Paim, 20oo).

Por último, discute o significado das possíveis mudanças no processo de planejamento e gestão das PPS 
tendo em vista a necessária elevação da capacidade de governo das organizações públicas em face da complexidade da situação de saúde da população e do momento atual em termos da política e gestão do sistema de saúde, no qual se avizinha uma renovação no universo de prefeitos e secretários municipais de saúde.

\section{Políticas Públicas Saudáveis: definições e conceitos}

A concepção atual de Promoção da Saúde está associada a um conjunto de valores (vida, saúde, solidariedade, eqüidade, democracia, cidadania, desenvolvimento sustentável, participação e parceria), referindo-se a uma "combinação de estratégias que envolvem a ação do Estado (políticas públicas saudáveis), da comunidade (reforço da ação comunitária), de indivíduos (desenvolvimento de habilidades pessoais), do sistema de saúde (reorientação do sistema de saúde) e de parcerias interinstitucionais, trabalhando com a noção de responsabilização múltipla, seja pelos problemas, seja pelas soluções propostas para os mesmos" (Buss, 2003, p. 16).

No que diz respeito à noção de PPS, o debate que vem sendo desenvolvido nas conferências internacionais destaca a vinculação entre as políticas sociais e econômicas, expressa a necessidade de assegurar a participação democrática no processo de formulação de políticas, chama a atenção para a responsabilização compartilhada entre o setor público e o setor privado, incorpora a proposta de estabelecimento de parcerias entre os diversos setores e enfatiza a capacidade de as pessoas e grupos se mobilizarem e se organizarem para o desencadeamento de ações políticas coletivas voltadas à intervenção sobre os determinantes da saúde em diferentes contextos e territórios (Buss, 2003).

Segundo Buss (2003, p. 27), as políticas públicas saudáveis se expressam por diversas abordagens complementares, que incluem legislação, medidas fiscais, taxações e mudanças organizacionais, e por ações coordenadas que apontam para a eqüidade em saúde, a distribuição mais eqüitativa da renda e políticas sociais. Essa compreensão abrangente do termo representa, assim, uma superação da perspectiva que marcava o entendimento anterior do alcance da promoção da saúde, limitado à correção de comportamentos individuais, isto é, à mudança dos "estilos de vida" prejudiciais à saúde.

De fato, a incorporação do termo "saudável" à expressão "políticas públicas" abre uma "janela de oportunidade" (Kingdon, 1995) do ponto de vista da reflexão crítica no campo das políticas, na medida em que aponta a possibilidade de incorporar ao debate acerca dos objetivos a serem perseguidos e das estratégias a serem implementadas o avanço conceitual que vem ocorrendo no âmbito da Saúde Coletiva, especialmente no que diz respeito aos estudos produzidos na área de Epidemiologia acerca da situação de saúde da população e seus determinantes e à reflexão que vem sendo realizada na área de Planejamento e Gestão em saúde acerca dos diversos enfoques e opções metodológicas para a formulação e implementação de políticas e ações de saúde.

No que se refere à contribuição da Epidemiologia, o debate atual em torno de temas como transição epidemiológica e suas características específicas na sociedade brasileira (Barreto e Hage, 200o), os estudos sobre os determinantes e formas de expressão das desigualdades sociais em saúde (Barreto, 1998; Almeida Filho, 2001), a problematização da noção de saúde, "ponto cego da Epidemiologia" (Almeida Filho, 1999), e a possibilidade de sua conceituação positiva (Almeida Filho e Andrade, 2003), bem como a reflexão sobre a noção de risco (Caponi, 2003; Castiel, 2003) e os estudos sobre vulnerabilidade (Ayres, 2002; Ayres, 2003), aliados ao desenvolvimento dos sistemas informatizados de geração de dados sobre condições de vida e saúde dos diversos grupos populacionais, constituem um "solo" epistemológico e tecnológico em que pode vir a se reconfigurar o objeto de conhecimento e intervenção no âmbito das políticas públicas.

Já na área de Planejamento e Gestão em saúde, a crítica ao enfoque normativo, o desenvolvimento do enfoque estratégico-situacional e, sobretudo, o enriquecimento desse enfoque a partir do diálogo entre as distintas correntes de pensamento na área (Rivera e Artmann, 1999; Rivera, 2001; Rivera, 2003) e destas com a Epidemiologia (Teixeira, 2003b) subsidiam o desenvolvimento de processos de formulação de políticas, planejamento e execução de ações que podem vir a privilegiar a intersetorialidade das políticas, a integralidade das práticas e a participação social na gestão dos planos, programas e projetos de ação, incorporando métodos, técnicas e instrumentos que 
subsidiem a tomada de decisões de forma coerente com os princípios e valores ético-políticos incorporados aos movimentos da promoção da saúde e da reforma sanitária brasileira.

Nessa perspectiva, vários autores chamam a atenção para a importância de trabalhar com um enfoque por problemas, desenvolvendo a análise dos seus determinantes estruturais e formas de expressão fenomenológica em termos de condições de vida e saúde dos diversos grupos sociais, identificados a partir do geoprocessamento de informações que permitem o mapeamento de condições de vida e saúde da população em distintos territórios (Castellanos, 1997; Paim, 1997).

Do ponto de vista da seleção e implementação de alternativas para o enfrentamento dos problemas identificados, a reflexão crítica sobre as políticas vigentes e a experimentação de práticas alternativas, que incorporam a tentativa de integração de ações e serviços aliada à mobilização social e comunitária na busca de soluções aos problemas e na execução de ações, têm gerado, inclusive, a atualização da reflexão sobre a proposta de Vigilância da Saúde (Teixeira, 2001; Teixeira, 2002; Freitas, 2003; Paim, 2003), que incorpora, desde suas origens, a noção de Políticas Públicas Saudáveis (Paim, 1994).

Do mesmo modo, a noção de "cidades saudáveis" (Westphal, 1997; Sperandio, 2004), expressão das PPS no âmbito da gestão governamental das cidades, pode vir a se beneficiar de um diálogo construtivo com os saberes e práticas que vêm sendo desenvolvidos no campo da Saúde Coletiva, as quais dão conteúdo a idéias como "promoção da cidadania" e o envolvimento de organizações “comunitárias”, recobertas no discurso original do movimento da Promoção da Saúde pela noção de empowerment (Carvalho, 2004).

A adoção da proposta de criação de "cidades saudáveis" pode ser entendida, assim, como uma importante inovação conceitual e organizacional no âmbito da administração pública municipal, apontando para a necessária superação da fragmentação de objetos e práticas de intervenção, tão bem denunciada por Matus (1996) quando comenta que "o governo tem setores porém a realidade tem problemas”, chamando a atenção para a necessidade de uma mudança radical no modo de governar situações complexas, como as que são enfrentadas no cotidiano das cidades hoje.

\section{Planejamento e Gestão de Políticas Públicas Saudáveis}

O planejamento e a gestão de PPS èm nível nacional e estadual ou, particularmente, em nível municipal podem vir a ser um espaço privilegiado para a adoção de conceitos e experimentação de métodos, técnicas e instrumentos que incorporem a interdisciplinaridade na análise dos problemas, a intersetorialidade na definição das soluções e a horizontalidade (Santos, 2003) na implementação de ações voltadas para a mudança e transformação das condições de vida e saúde da população, de acordo com suas necessidades sociais e demandas políticas.

Apesar da existência de um relativo consenso entre estudiosos e gestores públicos com relação ao caráter virtuoso da descentralização, da intersetorialidade e da busca de modelos de gestão alternativos (Junqueira, 1998; Siqueira, 1998), persistem dúvidas quanto às formas mais adequadas de planejar, organizar, conduzir, gerir e avaliar tais intervenções (Viana, 1998). Nesse particular, há divergências quanto à ênfase e à precedência de reformas administrativas para compor novos arranjos organizacionais e institucionais, além de perplexidades em relação às práticas políticas necessárias a uma redistribuição do poder e à reorganização dos processos de trabalho (Paim, 1992; Inojosa, 1998) visando formular e implementar políticas e assegurar efetividade à ação intersetorial. O "loteamento" de cargos do governo entre diferentes tendências e grupos políticos (Inojosa, 1998), o aprisionamento da política pelos interesses das elites locais, o burocratismo, o corporativismo e os limites impostos pela política econômica (Viana, 1998) representam constrangimentos que devem ser considerados no desenho estratégico.

Um dos desafios colocados por esse processo diz respeito ao planejamento, organização e gerenciamento da ação intersetorial, isto é, da conjugação dos esforços de distintos setores, como saúde, educação, saneamento, segurança, transporte, habitação, entre outros, visando a racionalização dos recursos existentes e a transformação das atividades desenvolvidas tendo em vista maior efetividade e impacto sobre os problemas e necessidades sociais. Alguns autores (Suárez, 1992; Junqueira, 1998; Inojosa, 1998) recomendam partir dos problemas da população de um 
dado território e buscar as soluções articulando distintas políticas e setores. Assim, "as ações intersetoriais dos serviços urbanos exigem um planejamento que garanta a integração dos objetivos e que esteja em constante processo de aprendizagem, no sentido de ser voltado à realidade altamente dinâmica e mutante de nossa sociedade" (Siqueira, 1998, p.105).

Com efeito, a formulação e a implementação de propostas inovadoras, como é o caso das Políticas Públicas Saudáveis, exigem o desenvolvimento de um processo de planejamento e programação que constitua um espaço de poder compartilhado e de articulação de interesses, saberes e práticas das diversas organizações envolvidas. A escolha de um determinado enfoque teórico-metodológico a ser utilizado no processo de formulação de políticas públicas e de planejamento de ações intersetoriais deve levar em conta, portanto, a necessidade de coerência entre os propósitos definidos e os métodos selecionados (Testa, 1992), de modo a permitir o desencadeamento de um processo de reorganização das práticas gerenciais, dos formatos organizacionais e, sobretudo, dos processos de trabalho no âmbito das instituições envolvidas (Paim, 1993; Schraiber, 1995; Schraiber e col, 1999).

Dando continuidade à reflexão desenvolvida a partir de uma experiência de capacitação da equipe multiprofissional responsável pelo planejamento das ações intersetoriais para a promoção da paz e combate à violência no município de Salvador (Teixeira e Paim, 200o), consideramos pertinente tomar como ponto de partida o enfoque situacional (Matus, 1993; 1997) para o planejamento e gestão de planos, programas e projetos de ação intersetorial (Suárez, 1993), particularmente no âmbito municipal e local, tratando de agregar algumas sugestões metodológicas inspiradas na revisão da literatura recente na área.

Os principais argumentos a favor desta opção derivam de características intrínsecas à concepção teórico-metodológica que fundamenta esse enfoque, quais sejam, a compreensão do objeto do planejamento como sendo constituído por problemas e oportunidades reais de intervenção, a concepção do processo de planejamento como sendo constituído de "momentos" que se interpenetram, a proposta de "modularização" do produto do planejamento e a "teoria de governo" embutida no enfoque que aponta em direção a uma ampliação da esfera pública, através da democratiza- ção das relações e de uma intensificação da ação comunicativa entre gestores, técnicos e usuários dos serviços (Teixeira e Paim, 20oo).

A incorporação do "enfoque por problema" supõe a identificação e seleção de problemas que podem ser analisados de distintos ângulos, como é o caso dos problemas de saúde, contribuindo para a identificação e articulação das ações a serem realizadas por cada instituição envolvida no seu enfrentamento e solução. A base teórico-metodológica dessa concepção reside no "fluxograma situacional” (Matus, 1993), instrumento de análise que permite uma abordagem multireferencial aos problemas de saúde, particularmente aqueles cuja determinação transborda o âmbito de ação do sistema de serviços de saúde. O desenvolvimento de processos de análise desse tipo de problema tanto possibilita o estabelecimento de um diálogo entre os sujeitos envolvidos quanto permite a identificação da contribuição que cada instituição ou "setor" pode vir a aportar na intervenção sobre seus determinantes e condicionantes (Teixeira e Paim, 200o).

No que diz respeito ao processo de planejamento cabe ressaltar que a compreensão dos diversos momentos que o constituem - o momento explicativo, o momento normativo, o momento estratégico e o momento tático-operacional - desafia o tempo cronológico e remete à pluralidade de tempos (técnico e político) e simultaneamente à pluralidade de dimensões do(s) sujeito(s) que planeja(m) e se conecta com a pluralidade de instituições que compõem os vários "setores" da ação governamental envolvidos em um esforço de planejamento intersetorial.

A noção de "momento" se articula dinamicamente com a proposta de organização do plano como uma estrutura "modular", que permite a definição de objetivos e o desenvolvimento de atividades e tarefas que podem estar situadas em tempos distintos do processo de intervenção sobre os problemas, ao mesmo tempo em que contempla a integração de sujeitos institucionais distintos, flexibilizando, na prática, estruturas organizativas burocráticas. Desse modo, pode ter como conseqüência a abertura de espaços de diálogos e negociação coletiva em torno do redirecionamento dos programas e projetos desenvolvidos, a realocacão dos recursos públicos e o incremento da participação social, fundamentada na co-responsabilização pela promoção do bem-estar coletivo, contribuindo para o 
processo de acumulação de conhecimentos e de poder por parte dos diversos sujeitos institucionais e atores sociais envolvidos no processo.

Pelo exposto, a formulação e implementação de PPS podem assumir, como referência, os conhecimentos acumulados acerca das características e dos determinantes dos problemas de saúde em diversos níveis e os valores éticos e políticos dos distintos sujeitos, adequando métodos, técnicas e instrumentos de acordo com as finalidades das organizações (governamentais ou não-governamentais) onde o processo de planificação for desenvolvido e o contexto onde estas estejam atuando.

Do ponto de vista metodológico, o "planejamento de políticas públicas saudáveis" pode levar em conta os diversos momentos sugeridos no enfoque situacional, desdobrados de acordo com as necessidades e demandas dos atores envolvidos, o que supõe o mapeamento dos atores e de suas posições diante dos problemas e soluções apresentadas ao debate, de modo que possa se estabelecer um processo dinâmico e flexível de negociação e pactuação em torno de compromissos, responsabilidades e expectativas de cada um deles.

A operacionalização de um processo dessa natureza ao nível nacional, estadual ou municipal requer, por parte da equipe responsável pela condução do processo de formulação e implementação das PPS, além da definição clara dos propósitos - conservação, mudança ou transformação do conteúdo e da forma de organização das práticas de saúde, recriação dos métodos de planejamento e gestão, de modo a incorporar e adaptar as propostas metodológicas disponíveis ao contexto político e institucional onde seja desencadeado esse processo (Teixeira, 2001; 2003).

Esse processo pode contemplar a montagem de uma base técnica-operacional para a análise permanente da situação de saúde, em territórios definidos (municípios, microrregiões, estados, macrorregiões e o próprio país), ponto de partida para a construção de cenários futuros que emoldurem a tomada de decisão, a formulação de políticas (Situação - objetivo em função do tempo político disponível) e a definição de prioridades. Isto resulta, em outros termos, na construção da Agenda do governo com relação à Saúde, definida a partir da seleção de problemas, determinantes e/ou de pontos críticos que se constituirão em objeto de intervenção por parte das organizações públicas e seus parceiros (setor privado e comunidade organizada e atuante em espaços territoriais selecionados em função da distribuição social dos problemas e dos grupos prioritários).

O desenho e implementação das estratégias de ação, por sua vez, supõem a programação das operações e a seleção das tecnologias a serem utilizadas na execução das ações, as quais podem contemplar, de acordo com a natureza dos problemas selecionados, um amplo leque constituído por ações de promoção da saúde, prevenção de riscos e agravos, assistência e recuperação, definidas em função da disponibilidade de oferta e possibilidade de integração entre os serviços de saúde e as ações desenvolvidas por outros setores nos distintos territórios, tendo em vista a melhoria das condições de vida e trabalho e a elevação da capacidade (empowerment) de indivíduos e grupos com relação à manutenção da saúde e defesa da vida.

Finalmente, cabe destacar a importância de se estabelecer os critérios e indicadores que serão utilizados no processo de monitoramento e avaliação das ações realizadas e dos resultados alcançados, podendo-se, inclusive, definir-se uma "linha de base" que referencie a avaliação periódica dos progressos na implementação das políticas e permita a comparação dos resultados alcançados com a situação inicial, informações que podem, inclusive, ser úteis para marketing político, a revelar a eficiência e efetividade das intervenções governamentais, caso ocorram, contribuindo para a legitimação de projetos e práticas de governo.

\section{Considerações Finais}

A incorporação critica e a ressignificação da proposta de formulação e implementação de Políticas Públicas Saudáveis trazem consigo uma grande possibilidade de resgate dos ideais da Reforma Sanitária Brasileira, especialmente a adoção do conceito ampliado de Saúde como referencial para a identificação e análise dos problemas e das necessidades sociais de saúde da população, ponto de partida para a tomada de decisões e elaboração de planos, programas e projetos de ação.

Ao mesmo tempo, a incorporação dessa proposta, particularmente no âmbito municipal, reforça e potencializa as iniciativas de mudança nas estruturas e práticas de gestão governamental, criando condições 
favoráveis à modernização e profissionalização da gestão das organizações públicas em nosso meio, de modo a se poder perseguir o ideal de conjugar eficiência, efetividade e participação democrática na tomada de decisões e implementação das ações e serviços.

Institucionalizar o planejamento situacional, mudar a prática e a cultura organizacional, pressupõe e implica enfrentar o desafio de constituir sujeitos competentes tecnicamente, comprometidos politicamente e sensibilizados com os valores que animam o debate sobre a formulação e implementação de PPS, isto é, o direito a saúde, a defesa da vida, a luta pela justiça social e a ressignificação dos ideais de participação política e solidariedade social no contexto contemporâneo.

Isso reforça a necessidade do diálogo entre gestores e pesquisadores da área de políticas sociais e de saúde em particular, bem como a necessidade de investir em processos de capacitação e educação permanente de dirigentes e técnicos, não só das instituições de saúde, mas também das instituições envolvidas na elaboração e execução de políticas públicas de modo geral, inclusive programas e projetos que contemplem a implementação de ações voltadas ao enfrentamento de problemas cujos determinantes extrapolam o "espaço de governabilidade" do sistema de saúde.

De fato, a diversificação dos problemas e objetos de investigação e intervenção, a multiplicidade de abordagens desses problemas, demanda o estabelecimento de diálogos interdisciplinares e a ampliação do debate em torno de alternativas de política e ampliação do leque de ações possíveis para a promoção, proteção e preservação da saúde individual e coletiva, balizadas por valores éticos e políticos que dêem suporte a práticas consentâneas com a preservação e criação de "modos (saudáveis) de andar a vida”, núcleo central do projeto histórico da reforma sanitária brasileira.

\section{Referências}

AKERMAN, M. et al. A concepção de um projeto de observatório de qualidade de vida: relato de uma experiência realizada em Campinas-SP. Saúde e Sociedade, São Paulo, v. 6, n.2, p. 83-99, 1997.

ALMEIDA FILHO, N. O conceito de saúde: ponto cego da epidemiologia. Conferência proferida em concurso para Professor Titular de Epidemiologia do ISC-UFBA, 31 de julho de 1999, 25 p. (mimeo)
ALMEIDA FILHO, N.; ANDRADE, R. F. S. Halopatogênese: esboço de uma teoria geral de saúde-doença como base para a promoção da saúde. IN: CZERESNIA, D.; FREITAS, C. M. (Org.). Promoção da saúde: conceitos, reflexões, tendências. Rio de Janeiro: Editora FIOCRUZ, 2003. p. 97-115.

AYRES, J. R. C. M. Epidemiologia, promoção da saúde e o paradoxo do risco. Revista Brasileira de Epidemiologia, São Paulo, v. 5, supl. 1, p. 28-42, 2002.

AYRES, J. R. C. M. et al. O conceito de vulnerabilidade e as práticas de saúde: novas perspectivas e desafios. CZERESNIA, D.; FREITAS, C. M. (Org.) Promoção da Saúde: conceitos, reflexões, tendências. Rio de Janeiro: Editora FIOCRUZ, 2003. p. 117-139.

BARRETO, M. Por uma epidemiologia da saúde coletiva. Revista Brasileira de Epidemiologia, São Paulo, v. 2, n.2, p.194-222, 1998.

BARRETO, M; HAGE, E. Determinantes das condições de saúde e problemas prioritários no país. In: $C A$ DERNO da $11^{a}$ Conferencia Nacional de Saúde. Brasília: Ministério da Saúde, 200o. p. 235-259.

BRASIL. Ministério da Saúde. Projeto promoção da saúde: cartas e declarações das conferencias internacionais de promoção da saúde. Brasília, 2001. 55 p.

BRASIL. Ministério da Saúde. Agenda Nacional de Prioridades de Pesquisa em Saúde. Relatório de progresso. In: CONFERÊENCIA Nacional de Ciência, Tecnologia e Inovação em Saúde, 2.,mar. 2004, Brasília. Brasília, 2004. p. 83-164.

BUSS, P. M. Uma introdução ao conceito de promoção da saúde. In: CZERESNIA, D.; FREITAS, C. M. (Org.). Promoção da saúde: conceitos, reflexões, tendências. Rio de Janeiro: Editora FIOCRUZ, 2003. p. 15-38.

CAPONI, S. A saúde como abertura ao risco. In: CZERESNIA, D.; FREITAS, C. M. (Org.). Promoção da saúde: conceitos, reflexões, tendências. Rio de Janeiro: Editora FIOCRUZ, 2003. p. 55-77.

CARVALHO, S. R. Os múltiplos sentidos da categoria "empowerment" no projeto de promoção da saúde. Cadernos de Saúde Pública, Rio de Janeiro, v. 2o, n. 4, p.1088-1095, 2004.

CASTELLANOS, P. L. Epidemiologia, saúde pública, situação de saúde e condições de vida: considerações conceituais. In: BARATA, R. (Org.). Condições de vida e situação de saúde. Rio de Janeiro: ABRASCO, 1997. p. 31-75. 
CASTIEL, L. D. Dédalo e os Dédalos: identidade cultural, subjetividade e os riscos à saúde. In: CZERESNIA, D.; FREITAS, C. M. (Org.). Promoção da saúde: conceitos, reflexões, tendências. Rio de Janeiro: Editora FIOCRUZ, 2003. p.79-95.

FERRAZ, S. T. Cidades saudáveis: uma urbanidade para 200o. Brasília: Paralelo 15, 1999. 103 p.

FREITAS, C. M. A Vigilância da Saúde para a promoção da Saúde. In: CZERESNIA, D.; FREITAS, C. M. (Org.). Promoção da saúde: conceitos, reflexões, tendências. Rio de Janeiro: Editora FIOCRUZ, 2003. p. 141-159.

INOJOSA, R. M. Intersetorialidade e a configuração de um novo paradigma organizacional. Revista de Administração Pública, Rio de Janeiro, v. 32, n.12, p.35-48, 1998.

JUNQUEIRA, L. P. Novas formas de gestão na saúde: descentralização e intersetorialidade. Saúde e Sociedade, São Paulo, v. 6, n.2, p. 31-46, 1997.

JUNQUEIRA, L. P. Descentralização e intersetorialidade: a construção de um modelo de gestão municipal. RAP, Rio de Janeiro, v. 32, n. 2, p. 11-22, 1998.

KINGDON, J. W. Como chega o momento de uma idéia? In: AGENDAS alternativas e políticas publicas. Tradução de Alternatives agendas and public policies. Michigan: Addison-Wesley Educacional Publisher, 1995.

MATUS, C. Política, planificação e governo. 2. ed. Brasília: IPEA, 1993. 2 Tomos. p. 297-554.

MATUS, C. Chimpanzé, Maquiavel e Ghandi: estratégias políticas. São Paulo: FUNDAP, 1996. 294 p.

MATUS, C. Los 3 cinturones del gobierno: gestión, organización y reforma. Caracas: Fondo Editorial Altadir, 1997. $262 \mathrm{p}$.

PAIM, J. S. Burocracia y aparato estatal: implicación para la planificación y instrumentación de politicas de salud. In: TEIXEIRA, S. F. (Org.). Estado y politicas sociales en America Latina. Mexico: Universidad Autonoma Metroplitana, Universidad Xochimilco, 1992. p.293-311.

PAIM, J. S. A reorganização das práticas de saúde em distritos sanitários In: MENDES, E. V. (Org.) Distrito Sanitário: o processo social de mudança das práticas sanitárias do Sistema Único de Saúde. São Paulo: HUCITEC; Rio de Janeiro: ABRASCO, 1993.

PAIM, J. S. A Reforma Sanitária e os modelos assistenciais In: ROUQUAYROL, M. Z. Epidemiologia \& Saúde. Rio de Janeiro: MEDSI, 1994. p. 455-466.
PAIM, J. S. Abordagens teórico-conceituais em estudos de condições de vida e saúde: notas para reflexão e ação. In: BARATA, R. (Org.). Condições de vida e situação de saúde. Rio de Janeiro: ABRASCO, 1997. p. 7-30.

PAIM, J. S. Vigilância da saúde: dos modelos assistenciais para a promoção da saúde. In: CZERESNIA, D.; FREITAS, C. M. (Org.). Promoção da saúde: conceitos, reflexões, tendências. Rio de Janeiro: Editora FIOCRUZ, 2003. p. 161-174.

RIBEIRO, R C. A construção de um município saudável: descentralização e intersetorialidade: a experiência de Fortaleza. Saúde e Sociedade, São Paulo, v.6, n. 2, p. 47-54, 1997.

RIVERA, J. U. Reflexões sobre a subjetividade na gestão a partir do paradigma da organização que aprende. Ciência \& Saúde Coletiva, Rio de janeiro, v. 6 , n. 1, p. 209-219, 2001.

RIVERA, J. U. Cultura e liderança comunicativa. In: RIVERA, J. U. Análise estratégica em saúde e gestão pela escuta. Rio de Janeiro: Editora FIOCRUZ, 2003. p. 285-218.

RIVERA, J. U.; ARTMANN, E. Planejamento e gestão em saúde; flexibilidade metodológica e agir comunicativo. Ciência \& Saúde Coletiva, Rio de Janeiro, v. 4, n.2, p. 355-365, 1999.

SANTOS, M. Por uma outra globalização: do pensamento único a consciência universal. Rio de Janeiro: Record, 2003. $180 \mathrm{p}$.

SCHRAIBER, L. B. Políticas públicas e planejamento nas práticas de Saúde. Saúde em Debate, São Paulo, n. 47, p. 28-35, jun. 1995.

SCHRAIBER, L. B. et al. Planejamento, gestão e avaliação em saúde: identificando problemas. Ciência \& Saúde Coletiva, Rio de Janeiro, v. 4, n.2, p. 221242, 1999.

SIQUEIRA, M. M. Transetoriedade dos serviços urbanos: preâmbulos de discussão. Revista de Administração Pública, Rio de Janeiro, v. 32, n.12, p.93-107, 1998.

SPERANDIO, A. (Org.). O processo de construção da rede de municípios potencialmente saudáveis, Campinas: IPES Editorial, UNICAMP, 2004. 2 v.

SUÁREZ, J. Contribuicion al marco teorico para el desarrollo de la accion intersectorial en salud. Educación Medica y Salud, Washington, v. 26, n.2, p.225-241, 1992. 
SUÁREZ, J. Proposicion de un modelo para el estudio y desarrollo de la accion intersectorial en salud. Educación Medica y Salud, Washington, v. 27, n.1, p.61-81, 1993.

TEIXEIRA, C. F. O futuro da prevenção. Salvador,: Casa da Qualidade, ano 2001a. 115 p.

TEIXEIRA, C. F. Planejamento Municipal em Saúde. Salvador, CEPS, ISC-UFBa, 2001b. 79p.

TEIXEIRA, C. F. (Org.) Promoção e vigilância da saúde. Salvador, CEPS-ISC, 2002. $128 \mathrm{p}$.

TEIXEIRA, C. F. Epidemiologia e planejamento de saúde. In: ROUQUAYROL, M. Z.; ALMEIDA FILHO, N. Epidemiologia \& Saúde. 6a. ed. Rio de Janeiro: MEDSI, 2003. p. 515-531.

TEIXEIRA, C. F. A mudança do modelo de atenção a saúde no SUS: desatando nos e criando laços Saúde em Debate, Rio de Janeiro, v. 27, n. 65, p 257-277, 2003.
TEIXEIRA, C. F.; PAIM, J. S. Planejamento e programação das ações intersetoriais para a promoção da saúde e da qualidade de vida. Revista de Administração Pública, Rio de Janeiro, v. 34, p. 63-8o, 2000.

TESTA, M. Tendências em planejamento. In: PENSAR em Saúde. Porto Alegre: Artes Médicas/ABRASCO, 1992. p. 89-128

VIANA, A. L. Novos riscos, a cidade e a intersetorialidade das políticas públicas. Revista de Administração Pública, Rio de Janeiro, v. 32, n.12, p.23-33, 1998.

WESTPHAL, M. F. Municípios saudáveis: aspectos conceituais. Saúde e Sociedade, São Paulo, v. 6, n.2, p. 9-18, 1997. 